\title{
Correlation for Length of Impinging Shock Induced Large Separation Bubble at Hypersonic Speed
}

\author{
R. Sriram ${ }^{1}$ and G. Jagadeesh ${ }^{2}$ \\ Indian Institute of Science, Bangalore, India, 560012 \\ Nomenclature \\ $C=$ Chapman-Rubesin constant \\ $h=$ specific enthalpy of flow, $\mathrm{MJ} / \mathrm{kg}$ \\ $L_{\text {sep }}=$ separation length, $\mathrm{mm}$ \\ $M \quad=\quad$ Mach number \\ $p \quad=\quad$ pressure, $\mathrm{Pa}$ \\ $R e \quad=\quad$ Reynolds numbere \\ $T=$ Temperature, $\mathrm{K}$ \\ $x=$ distance from the leading edge of the flat plate, $\mathrm{mm}$ \\ $\delta^{*} \quad=\quad$ boundary layer displacement thickness, $\mathrm{mm}$ \\ Common subscripts \\ $0=$ stagnation conditions \\ $1=$ Conditions immediately upstream of interaction \\ $3=$ conditions after interaction (in [10]) \\ $i=$ conditions at shock impingement location \\ $r=$ conditions at reattachment \\ $x=$ conditions at distance $\mathrm{x}$ from leading edge \\ $\infty=$ freestream conditions
}

\footnotetext{
${ }^{1}$ Postdoctoral Fellow, Mechanical Engineering, Physical Sciences and Engineering Division, King Abdullah University of Science and Technology, Thuwal, Saudi Arabia.

${ }^{2}$ Professor, Department of Aerospace Engineering, Indian Institute of Science, Bangalore- 12., and AIAA Member.
} 


\section{Introduction}

Interaction of boundary layer with a shock of sufficient strength may lead to flow separation (termed strong interaction) [1]. While the understanding of the shock wave boundary layer interaction (SBLI) phenomena has substantially grown at supersonic speeds especially due to sophisticated diagnostics and computations, many complications at hypersonic speeds are yet to be resolved. In order that the moderate to high total enthalpies associated with hypersonic flows are experimentally simulated, short duration impulse facilities like shock tunnels are required. There are however, few shock tunnel studies reported in the literature on hypersonic SBLI, over compression corners, generally with separation bubbles of lengths comparable with the boundary layer thicknesses [2-4].

The other fundamental case of impinging shock wave boundary layer interaction is particularly important at hypersonic speeds due to their occurrence in scramjet intakes, at off-design operation (at higher Mach number condition), when the ramp shock impinges on the cowl plate at some distance downstream of the leading edge. Such a case with the occurrence of a separation bubble is illustrated by Mahapatra and Jagadeesh (2009) [5]. It may be noted that, in such a case, the length of the separation bubble is comparable to (or nearly the same as) the distance of shock impingement from the leading edge. Separation bubbles of such lengths are termed large separation bubbles in the present study; i.e., for the case of impinging shock interaction, the ratio of separation length (distance from separation to reattachment location) to the distance of shock impingement location from the leading edge is of order

1. Though their occurrence may be sensed, there is hardly any focussed study on shock induced large separation bubble, reported in the literature. Often, the separation occurs very close to the leading edge, where the boundary layer is insignificantly thin. Studies on leading edge separation at hypersonic high enthalpy conditions have only been initiated in recent years [6].

Separation length is indeed a very important characteristic of strong interactions along with other quantities such as pressure and heat transfer rate distributions; it is often the reduction of the separation length, which is the objective of the control of the interaction. A number of similarity laws are reported in the literature, which relates separation length to the freestream conditions and other parameters such as incipient separation pressure/ plateau pressure and reattachment pressure. These include compression ramp/double wedge cases at supersonic [7] as well 
as hypersonic freestreams $[3,8]$, supersonic impinging shock cases $[9,10]$; the separation length for a hypersonic double cone [11] was also found to follow a correlation similar to that proposed by Davis and Sturtevant [3] for double wedges. Recently, John and Kulkarni [12] analyzed the important correlations reported in the literature, by comparing their numerical data for compression corner for Mach numbers ranging from 5 to 11.63 (the correlation [10] for impinging shock case was also compared by suitably adapting the parameters); it was noted that the correlations were not universal. Further, none of the correlations address large separation bubbles. It is with this backdrop that the present shock tunnel experiments on strong impinging shock wave boundary layer interactions with the generation of large separation bubbles were initiated. A correlation for separation length, obtained based on the experimental data at various freestream conditions and shock impingement locations, shall be presented.

\section{Shock Tunnel Experiments}

Experiments are performed in IISc hypersonic shock tunnel HST-2 [5]. It is a conventional shock tunnel where the high pressure maintained in the driver section (with driver gas helium) ruptures a metal diaphragm, generating a shock propagating in the driven section (with test gas air maintained at relatively low pressure- 500 mbar in the present study). On the reflection of the shock at the shock tube end, the test gas is compressed to high pressure and temperature (thus simulating the required high enthalpy), which subsequently expands through nozzle into the test section to the required Mach number (based on the nozzle area ratio). The enthalpy is varied by varying the diaphragm thickness, which varies the driver pressure (40 bar for low enthalpy cases and 60 bar for $2.4 \mathrm{MJ} / \mathrm{kg}$ case) [13]. The three different freestream conditions at which the experiments are performed, estimated from the pressure (and shock speed) measurements at the shock tube end and the pitot pressure measurement inside the test section, are given in Table 1. Schlieren flow visualizations obtained using high speed camera (Phantom V-310) and surface pressure measurements using fast response sensors (kulite sensors with uncertainty $\pm 0.5 \%$, PCB sensors with uncertainty $\pm 1 \%$ and MEMS sensors being developed in-house) are the flow diagnostics used for the investigation.

The strong impinging shock is generated by a wedge (or shock generator) of angle $31^{0}$ to the freestream. The wedge generates shocks of strengths (pressure ratio across the shock) 18.5 and $~ 35$ and angles with the freestream of $42.2^{0}$ and $\sim 40.2^{0}$ at Mach numbers 5.96 and $\sim 8.5$ respectively. The shock impinges on the surface of a flat plate (made of Hylem), placed $60 \mathrm{~mm}$ bellow the bottom of the wedge. A schematic of the test model (with the wedge and 
flat plate held by fixture which mounts them in the tunnel) is shown in Fig. 1. The flat plate can be moved forward or backward on the fixture by which the location of shock impingement may be varied.

For Mach 5.96 case, from inviscid oblique shock calculations, it is estimated that the shock impinges at a location of $90 \mathrm{~mm}$ from the leading edge, when the leading edge of the plate is placed $10 \mathrm{~mm}$ behind (in downstream direction) the leading edge of the shock generator. However, due to the interaction with the expansion fan from the rear end of the wedge, the shock gets turned (and weakened as well) a little, such that the point of impingement is little downstream of the location predicted by oblique shock calculations. From Euler computations (using the commercial code FLUENT, 2-D Steady simulations were performed to second order accuracy with upwind spatial discretization, employing density based implicit formulation with Roe flux differencing scheme and with least square cell-based method for gradient computation [13]) it was predicted that the shock impingement location was $5 \mathrm{~mm}$ downstream of the predictions from oblique shock calculations; i.e. at $95 \mathrm{~mm}$ from the leading edge rather. Thus the location of inviscid shock impingement is fixed in the present study, taking into account the interaction of the expansion waves from the rear end of the wedge with the impinging shock. The shock impingement location is varied from $55 \mathrm{~mm}$ from the leading edge to $100 \mathrm{~mm}$ from the leading edge. Further, although Mach reflection (similar to Type 2 [14]) is expected on the surface (for inviscid case) for all the Mach numbers, due to interaction with the expansion waves the Euler simulations always showed regular reflection (similar to Type 1[14]). Since the wedge angle is fixed, the shock strength is not varied in the present study independent of Mach number. However, the variation of Mach number naturally leads to different ranges of reattachment pressure (particularly the ratio of pressure at reattachment to the freestream pressure), due to the increasing shock strength with the Mach number.

Typical schlieren images of the flow fields at Mach 5.96 and 8.67 are shown in Fig. 2 (with inviscid shock impingement at $95 \mathrm{~mm}$ and $100 \mathrm{~mm}$ respectively from the leading edge). It is apparent that the length of the separation bubble is as much as the distance of shock impingement from the leading edge at Mach 5.96; even at Mach 8.67, they are comparable. The corresponding surface pressure distributions are shown in Fig. 3, with the schlieren images cut to scale (in the direction along the plate) also superimposed above. Due to the lack of thickness, it was not possible to mount pressure sensors close to the leading edge. Thus only for the Mach 8.67 case, it was 
possible to measure the pressure at the separation location (of $38 \mathrm{~mm}$ from the leading edge), which is $640 \mathrm{~Pa}$ (Fig. 3b). From free-interaction theory [1] the separation pressure is estimated to be $530 \mathrm{~Pa}$ assuming adiabatic wall, whereas for an isothermal cold wall (at $300 \mathrm{~K}$ ) the estimate was just $400 \mathrm{~Pa}$ [13]. The isothermal wall assumption also under-predicts by a large margin the plateau pressure; the plateau pressure for adiabatic wall was $730 \mathrm{~Pa}$ while that for isothermal wall was $500 \mathrm{~Pa}$, which can be compared with the pressures measured in the three locations immediately downstream of separation. Within the short run time of the shock tunnel the wall temperatures are not expected to change significantly, despite which the adiabatic wall estimates are apparently closer to the experiments. It can be suggested that the retarded fluid in the boundary layer, a little away from wall, is at high temperatures comparable to the adiabatic wall temperature and the short test times are not sufficient to rise the wall temperature; which can affect the velocity profiles as well. However, it is not possible to experimentally ascertain this, due to the lack of the measurement of boundary layer profiles for the simulated flow in the short duration facility.

In order to estimate the separation length, the location of separation and reattachment must be known. Since the upstream influence lengths (the distance upstream of separation till which the increase in pressure due to separation is felt in the boundary layer) are of the order of few displacement thicknesses, and in the present cases the boundary layers at the onset of the interactions are fairly thin (close to the leading edge), the separation location may be taken as the intercept of the separation shock on the flat plate surface. In order to obtain this location, the intensities along closely spaced vertical lines (crossing through the separation shock) are scanned. The coordinate locations of local intensity peaks corresponding to separation shock are noted and a line is fit along the points, whose intercept on the plate surface is noted. For the above mentioned case (at Mach 5.96, inviscid shock impingement at $95 \mathrm{~mm}$ ) it is found to be $16 \mathrm{~mm}$ from the leading edge, with uncertainty of $\pm 0.05 \mathrm{~mm}$ (based on the point at maximum distance from line fit) [13]. The reattachment is also located approximately from schlieren images, from the foot of the reattachment shock. It can be seen from the schlieren images (in Fig. 2) that the foot of the reattachment shock is detached but very close and almost parallel to the plate surface. Scanning the intensity along the foot of the shock close (and parallel) to the surface, a region of high intensity was observed for a maximum distance of $\sim 5 \mathrm{~mm}$ (for Mach 5.96 case shown in Fig. 2a; the foot was shorter for other cases). By placing the closely packed array of MEMS sensors (with $3 \mathrm{~mm}$ spacing between the centres of two subsequent sensors) in the apparent reattachment zone, the surface pressure measurements in the reattachment zone was spatially well resolved. The location of the 
measured peak pressure is taken as the reattachment location [13]. Thus, based on the maximum distance between the edge of the subsequently placed PCB and MEMS sensors in the reattachment region, the reattachment is located with an error of $\pm 2.5 \mathrm{~mm}$. For the above mentioned case the reattachment is located at $90.5 \mathrm{~mm}$ from the leading edge, at which location the peak pressure is measured; while the good correspondence between the measured peak pressure and the foot of the reattachment shock apparent in the superposed schlieren images can be noted. Thus the separation length for the above mentioned case is estimated to be $\sim 75 \mathrm{~mm}$. Similarly, at the same Mach number, for other shock impingement locations of $75 \mathrm{~mm}$ and $55 \mathrm{~mm}$ from the leading edge, the separation lengths are estimated to be $60 \mathrm{~mm}$ and $45 \mathrm{~mm}$ respectively, with the location of separation moving a little further closer to the leading edge with decrease in distance of shock impingement (10 $\mathrm{mm}$ and $5 \mathrm{~mm}$ respectively).

For the higher Mach number cases though the shock strengths (and correspondingly the measured surface pressures as well, including peak pressure) were more than twice that at Mach 5.96, it was observed that the separation length was substantially lesser than the Mach 5.96 cases with nearly same shock impingement location. For the case of Mach 8.67 flow with inviscid shock impingement location at $100 \mathrm{~mm}$ from the leading edge, the separation length was observed to be $\sim 60 \mathrm{~mm}$, with the location of separation at $\sim 38 \mathrm{~mm}$ from the leading edge, considerably downstream of Mach 5.96 cases. With inviscid shock impingement location of $80 \mathrm{~mm}$ from the leading edge the separation length was found to be $\sim 55 \mathrm{~mm}$ at Mach 8.67. For the higher enthalpy case $(2.4 \mathrm{MJ} / \mathrm{kg})$ at a very similar range of Mach number (8.21), the separation length was $\sim 70 \mathrm{~mm}$ with inviscid shock impingement location of $100 \mathrm{~mm}$ from the leading edge; $10 \mathrm{~mm}$ more than that at Mach 8.67 at lower enthalpy. However for both the enthalpies at the higher Mach number, the ratio of measured reattachment pressure to the freestream pressure was nearly the same.

It can be noted that the reattachment locations are nearly the same as, or sometimes upstream of, the estimated inviscid shock impingement locations. In fact, an interesting observed feature of these interactions is that the separation bubbles are 'taller and are biased upstream of the impingement location', as noted by Krishnan et al [15] in their numerical simulations for higher impinging shock strength, even at the ratio of reattachment pressure to the freestream pressure of 4.39. In the present study, the minimum pressure ratio is $\sim 30$ at Mach 5.96, and at Mach $~ 8.5$ the ratio is still higher, $\sim 70$. It must also be admitted that due to the boundary layer displacement effects at the 
wedge the actual location of shock impingement would be little upstream of the inviscid predictions from Euler simulations, the extent of which cannot however be ascertained. It is thus apparent that after interacting with the impinging shock, the separated shear layers reattach at distances relatively short ( $20 \mathrm{~mm}$ from the streamwise location of shock impingement on shear layer) compared to the separation bubble sizes; within this distance there is also the contribution of the expansion fan from the rear end of the wedge in postponing the reattachment location.

The expansion fan from the rear end of the wedge thus has three effects on the interaction: first, it weakens the impinging shock, reducing relatively the shock strength at impingement location. As far as the boundary layer is concerned, it essentially responds to the imposed adverse pressure at shock impingement, which is effectively due to the impinging shock of reduced strength (yet, despite which the reattachment pressure ratios are $\sim 30$ and above); this effect is better accounted by considering the measured reattachment pressure ratio, rather than considering the pressure ratio across the shock generated by the wedge (shock strength for the given wedge angle). Second, the expansion fan curves the impinging shock, due to which the impingement location is relatively downstream; and thirdly it interacts with the shear layer after it is turned by the impinging shock (though for a relatively short streamwise distance compared to the overall separation length). The two later effects do have direct consequences on the reattachment location; however small is the difference in the location due to the expansion when compared with the overall separation length. Measured separation length naturally takes into account the contribution of expansion fan in locating the reattachment. Hence, a phenomenological study of the relation of measured separation length to the measured reattachment pressure ratio is undertaken; the length scale based on shock impingement location is also obtained by considering the curving of the shock due to expansion, as mentioned before. Thus the role of expansion fan is accounted implicitly, such that the relation between the parameters considered in the present study (for large separation bubbles due to strong shocks) are applicable for different expansion fan strengths as well as for the cases without such expansion fan interactions.

\section{Note on boundary layer characteristics:}

The Reynolds numbers in the present experiments, defined based on the distance of shock impingement location from leading edge, range from $1 \times 10^{5}$ to $3.8 \times 10^{5}$. Thus, for all the cases, the boundary layer over the flat plate without shock impingement is expected to be laminar based on the correlation for transitional Reynolds number of 
hypersonic flows [16]. However, subsequent to flow separation the shear layer can become transitional; this cannot be substantiated from the present experimental data, and hence is not a concern of the present study. Another important consideration is the possible 3-dimensionality due to finite span of the model ( $80 \mathrm{~mm})$. While the addition of side fences to avoid spillage was adopted in earlier works, for low aspect ratio models the side fence invariably increases the separation length [17]. In the absence of side fences, Ball [18] suggested a conservative estimate of $10 \delta_{\mathrm{s}}$ from sides ( $\delta_{\mathrm{s}}$ is the boundary layer thickness at separation location), till which the three-dimensionality encroaches the separated flow field. For the Mach 5.96 cases when the separation is close to the leading edge, where the boundary layer thicknesses are estimated to be less than $1 \mathrm{~mm}$ (for adiabatic wall, based on van Driest [19]), the span of the model is more than $80 \delta_{\mathrm{s}}$. Even for the maximum boundary layer thickness at the separation location in the present set of experiments, of $\sim 3 \mathrm{~mm}$ for Mach $8.21 \mathrm{flow}$, the span of the model is $\sim 27 \delta_{\mathrm{s}}$. The present study thus concerns the 2-dimensional core around the spanwise centre.

\section{Correlation for Separation Length}

There are few correlations for separation length for the impinging shock interactions in the literature. The earlier correlation by Hakkinen (1959) [9] showed a complex relationship of separation length with Mach number, surface pressures as well as skin friction, but was experimentally verified only for Mach 2 flow. For laminar supersonic impinging shock boundary layer interaction, with separation lengths comparable to the local boundary layer displacement thickness (at the location of impingement), Katzer (1989) [10], based on numerical computations over adiabatic wall, proposed the following correlation for separation length.

$$
\frac{L_{s e p}}{\delta_{i}^{*}} \frac{M_{1}^{3}}{\sqrt{\operatorname{Re}_{x i} / C}}=4.4 \frac{p_{3}-p_{i n c}}{p_{1}}
$$

In the above correlation, the shock strength is given in relative terms (normalized by freestream pressure, or precisely, by the pressure immediately upstream of the interaction), given as the pressure in excess to the incipient separation pressure $p_{\text {inc }}$ (the minimum pressure required to cause flow separation), which can be found out from free interaction theory [1]. The incipient separation pressure may be evaluated, knowing freestream pressure, Mach number and importantly, the estimated value of skin friction coefficient at the location of impingement which essentially resists the imposed adverse pressure. The relation between the same correlation parameters is explored 
for the present study. The displacement thickness for hypersonic flows (at high Mach numbers, under the $\operatorname{limit} M_{\infty} \rightarrow \infty$ ) over the flat plate is given by:

$$
\delta^{*} \propto \frac{x}{\sqrt{\operatorname{Re}_{x} / C}} M_{\infty}^{2}
$$

Introducing this into the scaling for separation length in Eq. (1), the exploration is reduced to looking at the relation between $\frac{L_{s e p}}{x_{i}} M_{\infty}$ and $\frac{p_{r}-p_{i n c}}{p_{\infty}}$ for the present experimental data (for all cases), with the conditions immediately upstream of the interaction (denoted by the subscript 1) approximated as freestream conditions and the pressure after shock reflection (denoted by subscript 3) approximated by measured reattachment pressure. The relationship between the scaled separation length and the relative shock strength is plotted in Fig. 4 (which includes the data of all the cases, 2 experiments for each case).

It is evident that, since the separation bubble length is zero at incipient separation pressure (for any Mach number), the data at Mach 5.96 and at Mach 8.5 (including the higher enthalpy data) fall under two different lines. Similar behavior was also observed by Krishnan et al. [15], for their numerical data for impinging shock interactions, suggesting that for higher shock strengths (and larger separation bubbles) Katzer's correlation does not work.. It must however be admitted that in the present experiments the separation length naturally scales with the distance of shock impingement. Further, the considered shock strengths are such that the incipient separation pressure is insignificant. Typically the normalized reattachment pressure is $\sim 33.3$ at Mach 6 whereas the normalized incipient separation pressure is 1.5 ; at Mach 8 they are around $\sim 67.5$ and 2.5 respectively. Thus it seems that the separation length may be directly correlated with normalized reattachment pressure, rather than in relative terms with incipient separation pressure. In the above relation it is only incipient separation pressure which takes viscous effects into account; and if it is neglected, the relationship turns out to be purely inviscid. Reflecting on the trend in separation length with Mach number, it is clear that for a relatively smaller increase in Mach number from $\sim 6$ to $\sim 8.5$, the separation bubble considerably decreases in size despite the fact that the normalized reattachment pressure at higher Mach number is more than twice that at lower Mach number. Further, despite the normalized reattachment 
pressure being nearly the same between the cases at Mach $8.67(1.6 \mathrm{MJ} / \mathrm{kg})$ and Mach $8.21(2.4 \mathrm{MJ} / \mathrm{kg})$, the separation bubble is nearly $10 \mathrm{~mm}$ larger for the higher enthalpy case (with a relatively lower Mach number). From these considerations, an exponent $(>1)$ is worked out for Mach number, while retaining the linear relation with pressure. The similarity law for the separation bubble length from the present study is thus the following:

$$
\frac{L_{\text {sep }}}{x_{i}} M_{\infty}^{3} \propto \frac{p_{r}}{p_{\infty}}
$$

Fig. 5 shows all the experimental data ( 2 experimental data points for each case) confirming with the linear relationship between $\frac{L_{\text {sep }}}{x_{i}} M_{\infty}^{3}$ and $\frac{p_{r}}{p_{\infty}}$, which is fit by the solid line shown in the figure, whose slope is 5.8. The line fit has a small positive intercept in the $\mathrm{x}$-axis; indeed, for lower pressure ratios (less than incipient separation pressure) there is no separation (zero separation length), which gives a physical explanation for the positive intercept (though the correlation does not address small pressure ratios). Clearly the correlation is inviscid. The separation length is independent of Reynolds number; which is understandable as the boundary layer thickness and incipient separation pressure are insignificant compared to the scales of the separation bubble. However, the inverse cubic relationship with Mach number suggested by Katzer [10] holds for the present experiments too. Other scaling laws for separation length reported in the literature, for different flow regimes (from supersonic to hypersonic, including real gas effects) and different Reynolds number ranges, have also shown inverse cubic variation with Mach number $[3,7,8]$. However, for larger Reynolds numbers, the similarity law for supersonic separation bubble, proposed by Burggraf [7] based on asymptotic theory, showed non-linear dependence on pressure. Other correlations at hypersonic speeds for compression corner/ double ramp flows have also shown non-linear dependence on pressure $[3,9]$. In particular, according to the similarity law for double ramp flow derived based on asymptotic theory by Davis and Sturtevant [3] with the inclusion of high temperature effects, the separation length scaled by the distance of the location of separation was proportional to the $3 / 2^{\text {th }}$ power of the differential pressure ratio (the ratio of the difference between the reattachment and plateau pressures to the pressure upstream of the interaction). For the present case, the application of asymptotic theory is not straight forward as the compression ramp case, since while integrating the shear stress along the dividing stream line, the impingement of the shock on the separated shear layer 
has to be accounted; further, due to this effect of shock impingement on separated shear layer, the similarity law followed by the impinging shock case can considerably differ from that for the compression ramp case. However, the experiments by Davis and Sturtevant [3], in contrast to the predictions of asymptotic theory, showed a linear dependence on the differential pressure ratio; in terms of the separation length scaled with the distance of the reattachment from leading edge, using which analogy can be drawn with the present study, the maximum scaled separation length in their experiments ( 0.5) comparable with (but less than) the minimum scaled separation length in the present study ( 0.6 at Mach 8.67 with shock impingement at $100 \mathrm{~mm}$ from leading edge).

All other correlations in the literature deal with scaled separation lengths substantially smaller than that for the present study; for the separation bubble sizes considered in other correlations, the viscous terms are either directly involved by scaling the separation length with the characteristic scales of boundary layer, or indirectly through the pressure term which involves differential pressure (relative to incipient separation or plateau pressure, both of which are related to skin friction through free interaction theory). However, from the present experimental data, for impinging shocks of strength much greater than that required for incipient separation, the hypersonic large separation bubbles are found to follow an inviscid correlation, with a linear dependence of scaled separation length to the pressure ratio. Despite the moderate total enthalpies the role of specific heat ratio $\gamma$ cannot be addressed, since within the considered range of enthalpies the $\gamma$ does not vary significantly. Further, the real gas effects (especially arising due to flow dissociation) are not prominent even at $2.4 \mathrm{MJ} / \mathrm{kg}$ (the highest in the present study).

\section{Conclusion}

Shock tunnel experiments were performed to study the strong interaction between impinging shock (generated by a wedge of angle $31^{0}$ to the freestream) and boundary layer over a flat plate, with the formation of a large separation bubble of length comparable to the distance of shock impingement from the leading edge of the plate. For Mach numbers ranging from 5.96 to 8.67 , Reynolds numbers (based on shock impingement location) from $1 \times 10^{5}$ to $3.8 \times 10^{5} / \mathrm{m}$ and total enthalpy from $1.3 \mathrm{MJ} / \mathrm{kg}$ to $2.4 \mathrm{MJ} / \mathrm{kg}$, it was observed that the separation length followed a similarity law independent of the Reynolds number. According to the law, the separation length normalized by the distance of shock impingement from the leading edge was proportional to the ratio of reattachment pressure to the freestream pressure and inversely proportional to the cube of Mach number. 


\section{References}

[1] Delery, J., and Marvin, J. G., “Shock-wave boundary layer interactions,” AGARD-AG-280, 1986.

[2] Mallinson, S. G., Gai, S. L., and Mudford, N. R., "The interaction of a shock wave with a laminar boundary layer at a compression corner in high-enthalpy flows including real gas effects,” Journal of Fluid Mechanics, Vol. 342, 1997, pp. 1-35.

[3] Davis, J. -P., and Sturtevant, B., "Separation length in high-enthalpy shock/ boundary layer interaction," Physics of Fluids, Vol. 12, No. 10, 2000, pp. 2661-2687.

[4] Bleilebens, M., and Olivier, H., "On the influence of elevated surface temperatures on hypersonic shock wave/ boundary layer interaction at a heated ramp model," Shock Waves, Vol. 15, 2006, pp. 301-312.

[5] Mahapatra, D., and Jagadeesh, G., "Studies on unsteady shock interactions near a generic scramjet inlet," AIAA Journal, Vol. 47, No. 9, 2009, pp. 2223-2231.

[6] Moss, J. N., O’Byrne, S., Deepak, N. R., and Gai, S. L., "Simulations of hypersonic, high-enthalpy separated flow over a ‘tick' configuration,” AIP Conference Proceedinge, 1501, 1453, 2012.

[7] Burggraf, O. R., "Asymptotic theory of separation and reattachment of a laminar boundary layer on a compression ramp," AGARD-CP-168, 1975.

[8] Needham, D. A., "Laminar separation in hypersonic flow," Ph.D. Dissertation, University of London, 1965.

[9] Hakkinen, R. J., Greber, I., Trilling, L., and Abarbanel, S. S., "The interaction of an oblique shock wave with a laminar boundary layer,'” NASA TM 2-18-59W, 1959.

[10] Katzer, E., “On the length scales of laminar shock/ boundary-layer interaction,” Journal of Fluid Mechanics, Vol. 206, 1989, pp. 477-496.

[11] Swantek, A. B., and Austin, J. M., "Separation length scaling in hypervelocity double cone air flows," Proceedings of 28th International Symposium on Shock Waves, 2012, pp. 669-674.

[12] John, B., and Kulkarni, V., "Numerical assessment of correlations for shock wave boundary layer interaction," Computers and Fluids, Vol. 90, 2014, pp. 42-50.

[13] Sriram, R., "Shock tunnel investigations on hypersonic impinging shock wave boundary layer interaction," Ph. D. Dissertation, Indian Institute of Science, Bangalore, 2014.

[14] Edney, B. E., "Anomalous heat transfer and pressure distributions on blunt bodies at hypersonic speeds in the presence of impinging shock," The Aeronautical Research Institute of Sweden, FFA Report 115, 1968.

[15] Krishnan, L., Yao, Y., Sandham, N. D., and Roberts, G. T., "On the response of shock-induced separation bubble to small amplitude disturbances,” Modern Physics Letters B, Vol. 19, No. 28-29, 2005, pp. 67-70.

[16] Simeonides, G. A., "Correlation of laminar-turbulent transition data over flat plates in supersonic/hypersonic flow including leading edge bluntness effects," Shock Waves, Vol. 12, 2003, pp. 497-508. 
[17] Holden, M. S., "Boundary-layer displacement and leading-edge bluntness effects on attached and separated laminar boundary layers in compression corner. II- Experimental study,” AIAA Journal, Vol. 9, No. 1, 1971, pp. 84-93.

[18] Ball, K. O. W., "Flap span effects on boundary-layer separation,” AIAA Journal, Vol. 9, No. 10, 1971, pp. $2080-2081$.

[19] van Driest, E. R., "Investigation of laminar boundary layer in compressible fluids using the Crocco method," NACA TN2597, 1952.

\begin{tabular}{|c|c|c|c|c|}
\hline \multicolumn{7}{|c|}{ Table 1 Freestream conditions } \\
\hline $\begin{array}{c}\mathrm{M}_{\infty} \\
( \pm 3.2 \%)\end{array}$ & $\begin{array}{c}\mathrm{p}_{\infty}(\mathrm{Pa}) \\
( \pm 4.4 \%)\end{array}$ & $\begin{array}{c}\mathrm{T}_{\infty}(\mathrm{K}) \\
( \pm 4.4 \%)\end{array}$ & $\begin{array}{c}\operatorname{Re}_{\infty}(\mathrm{x} \mathrm{10} / \mathrm{m}) \\
( \pm 8.3 \%)\end{array}$ & $\begin{array}{c}\mathrm{h}_{0}(\mathrm{MJ} / \mathrm{kg}) \\
( \pm 3 \%)\end{array}$ \\
\hline 5.96 & 1277 & 160 & 4 & 1.3 \\
\hline 8.67 & 179 & 99 & 1.67 & 1.6 \\
\hline 8.21 & 254 & 168 & 1 & 2.4 \\
\hline
\end{tabular}

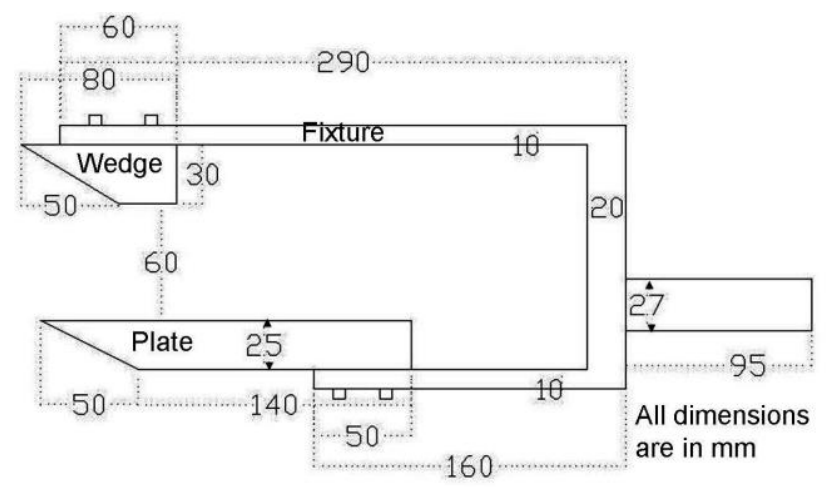

Fig. 1 Schematic of the test model. 


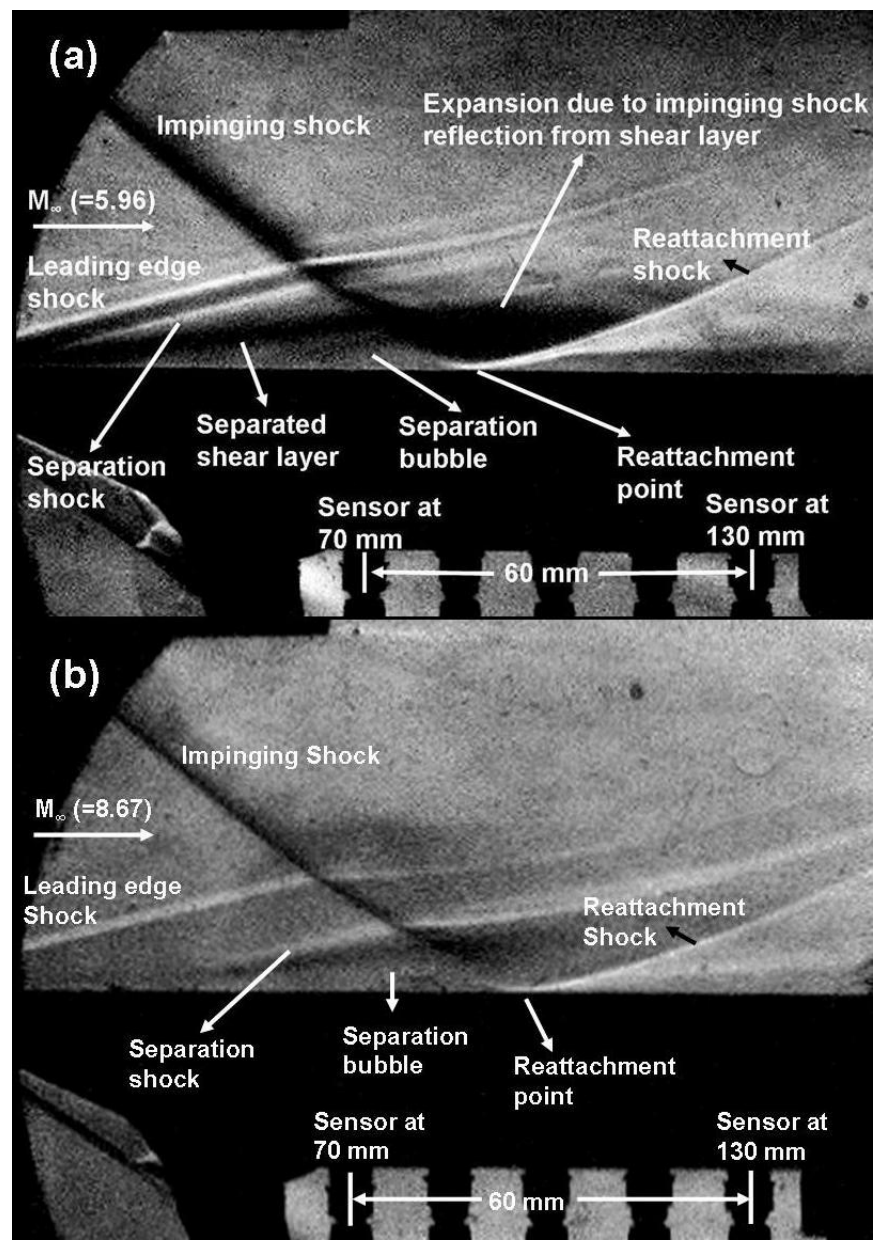

Fig. 2 Schlieren image of the flow fields (a) Mach 5.96 with inviscid shock impingement at $95 \mathrm{~mm}$ from the leading edge (b) Mach 8.67 with inviscid shock impingement at $100 \mathrm{~mm}$ from the leading edge. 

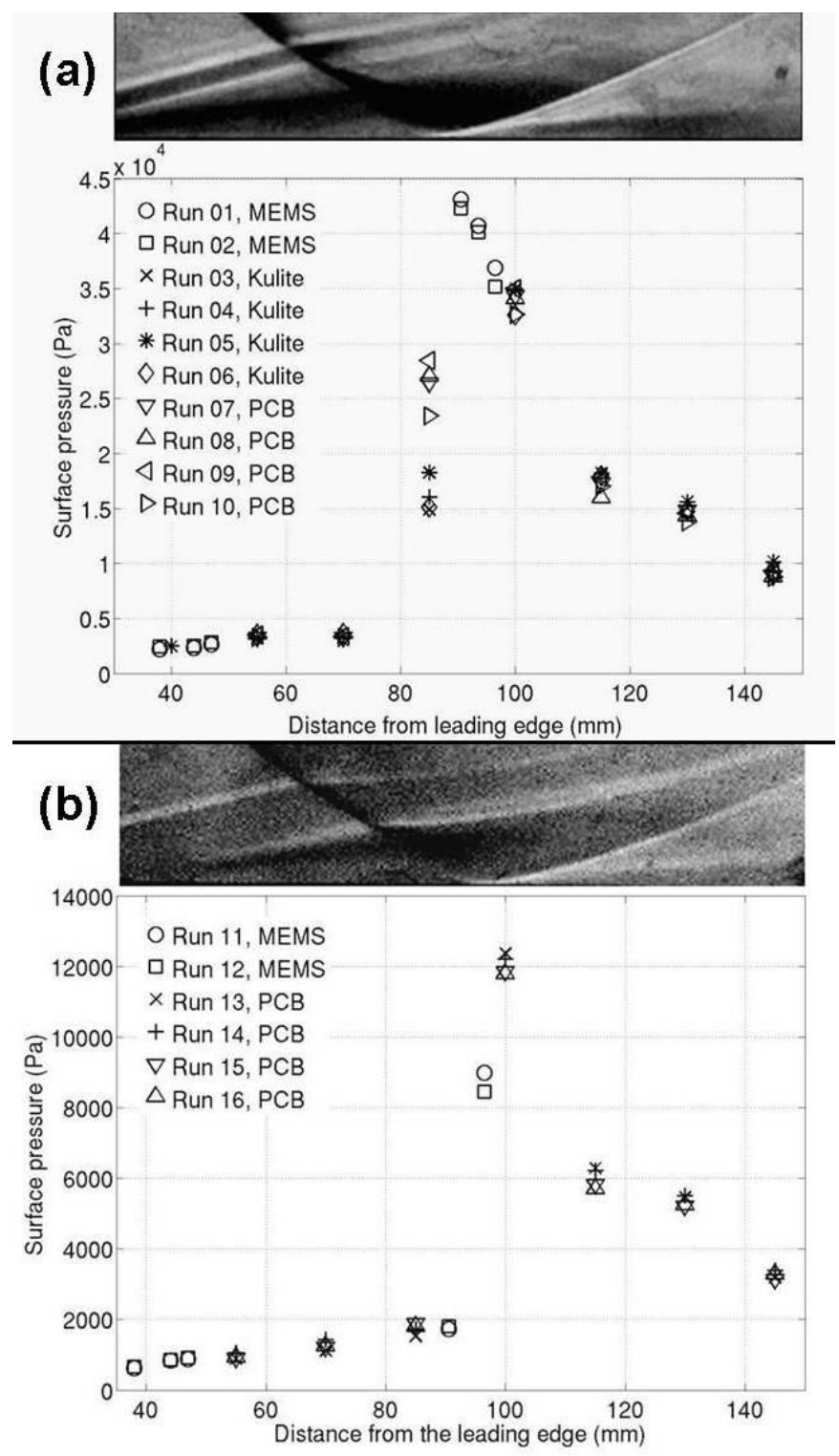

Fig. 3 Surface pressure distribution obtained from different sensors (a) Mach 5.96 with inviscid shock impingement at $95 \mathrm{~mm}$ from the leading edge (b) Mach 8.67 with inviscid shock impingement at $100 \mathrm{~mm}$ from the leading edge. 


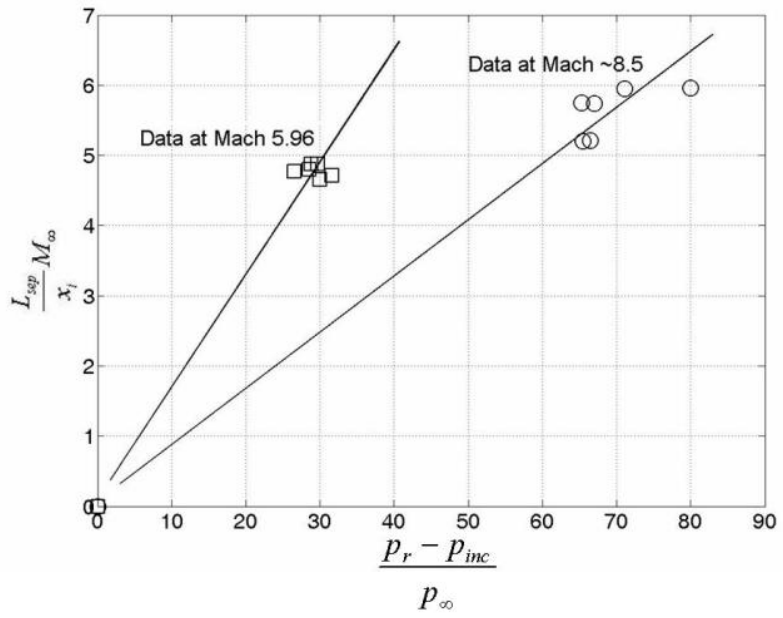

Fig. 4: Relationship between separation length, Mach number and shock strength (based on similarity law suggested by Katzer [10]).

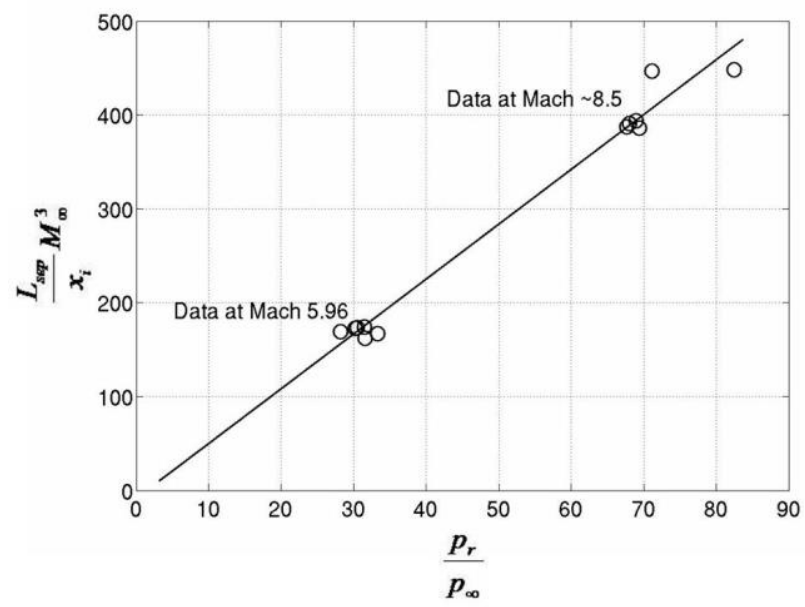

Fig. 5: Similarity law for length of large separation bubble induced by impinging shock. 